\title{
ENTIRE FUNCTIONS WHICH ARE INFINITELY INTEGER VALUED AT A FINITE NUMBER OF POINTS
}

\author{
BY
}

\author{
P. LOCKHART AND E. G. STRAUS
}

\begin{abstract}
This paper determines arithmetic limits for the growth rates of entire functions which are infinitely integer valued on a finite set $S$. The characterization of such functions with growth rate less than the arithmetic limit is complete if there exist exponential polynomials which are infinitely integer valued on $S$.
\end{abstract}

1. Introduction. Let $K$ be the rational field $\mathbf{Q}$ or an imaginary quadratic number field. An analytic function $f(z)$ is infinitely integer valued at a point $z_{0}$ if for all $n \geq 0, f^{(n)}\left(z_{0}\right)$ is an integer of $K$. In this note we continue the study of the family $\mathcal{F}$ of entire functions which are infinitely integer valued at a fixed set of points $z_{1}, z_{2}, \ldots, z_{s}$ of the complex plane.

Let $M(R, f)$ be the maximum modulus of $f(z)$ on $|z|=R$. Let $\rho, \sigma_{0}, \sigma_{1}, \ldots, \sigma_{k}$ be real numbers with $\rho>0, \sigma_{0}>0$. We say that $f(z)$ has growth rate $\left(\rho ; \sigma_{0}\right.$, $\left.\sigma_{1}, \ldots, \sigma_{k}\right)$ if for every $\varepsilon>0$,

$$
\log M(R, f)<R^{\rho}\left(\sigma_{0}+\frac{\sigma_{1}}{\log R}+\cdots+\frac{\sigma_{k}+\varepsilon}{(\log R)^{k}}\right)
$$

for all sufficiently large $R$, and

$$
\log M(R, f)>R^{\rho}\left(\sigma_{0}+\frac{\sigma_{1}}{\log R}+\cdots+\frac{\sigma_{k}-\varepsilon}{(\log R)^{k}}\right)
$$

for arbitrarily large $R$.

In this definition we allow the possibility that $k=-1$; then $f(z)$ has growth rate $(\rho)$ if and only if $f(z)$ has order $\rho$.

For each fixed $k$, we order the growth rates $\left(\rho ; \sigma_{0}, \ldots, \sigma_{k}\right)$ lexicographically; we do not compare growth rates with different values of $k$.

We call $\left(\rho ; \sigma_{0}, \ldots, \sigma_{k}\right)$ an arithmetic limit for $\mathcal{F}$ if

(i) $₹$ contains only countably many functions with growth rate less than $(\rho$; $\left.\sigma_{0}, \ldots, \sigma_{k}\right)$, and

(ii) $\mathcal{F}$ contains uncountably many functions with growth rate greater than $(\rho$; $\left.\sigma_{0}, \ldots, \sigma_{k}\right)$.

The work of E. G. Straus, D. Sato and A. M. Cayford $[\mathbf{2 , 3 , 7 , 9 ]}$ leads to the following.

THEOREM 1.1. For any $\left\{z_{1}, \ldots, z_{s}\right\}$ and any $\rho \geq s$, there exist $2^{\aleph_{0}}$ entire functions of order $\rho$ which are infinitely integer valued at $z_{1}, \ldots, z_{s}$. If there exists an entire function $f(z)$ of order $\rho<s$ which is infinitely integer valued at $z_{1}, \ldots, z_{s}$,

Received by the editors August 9, 1982 and, in revised form, March 25, 1985.

1980 Mathematics Subject Classification. Primary 30D15; Secondary 30D99. 
then $f(z)$ satisfies a linear differential equation with integral coefficients. In fact, $\rho=0$ or 1 and $f(z)$ is either a polynomial or an exponential polynomial, i.e. a Laurent polynomial in an exponential $e^{\lambda z}$.

Hence the arithmetic limit for the orders of functions in $\mathcal{F}$ is equal to $s$; in particular, it depends only on the number of points and not on their location.

The existence of a nontrivial function in $\mathcal{F}$ of order $<s$ places a severe restriction on the differences $z_{2}-z_{1}, \ldots, z_{s}-z_{1}$; In fact, the number of such $(s-1)$-tuples is denumerable and readily characterized.

In $[\mathbf{7}]$ the arithmetic limit $\left(\rho ; \sigma_{0}\right)$ for $\mathcal{F}$ is also determined. The result is as follows.

THEOREM 1.2. Let

$$
\sigma_{0}=\prod_{1 \leq i<j \leq s}\left|z_{i}-z_{j}\right|^{-2 / s},
$$

where $\sigma_{0}=1$ if $s=1$. Then there are $2^{\aleph_{0}}$ functions in $\mathcal{F}$ with growth rate $>\left(s ; \sigma_{0}\right)$, but only countably many functions in $\mathcal{F}$ with growth rate $<\left(s ; \sigma_{0}\right)$.

The characterization of functions in $\mathcal{F}$ with growth rate $<\left(s ; \sigma_{0}\right)$ is made more difficult by the fact that type limitations are not preserved under multiplication.

In $\S 2$ and $\S 3$ we determine the arithmetic limits $\left(\rho ; \sigma_{0}, \ldots, \sigma_{k}\right)$ for $\mathcal{F}$.

In $\S 4$ and $\S 5$ we discuss the case where $\mathcal{F}$ contains nonconstant functions of order $<s$.

2. Generalized Taylor series and growth rates of entire functions. Given a sequence $\varsigma_{1}, \varsigma_{2}, \ldots$, with $\left|\zeta_{n}\right| \leq r$ for all $n$, we can, as in $[\mathbf{2}]$, expand any entire function in the generalized Taylor series

$$
\begin{gathered}
f(z)=\sum_{n=0}^{\infty} a_{n}\left(z-\varsigma_{1}\right)\left(z-\varsigma_{2}\right) \cdots\left(z-\varsigma_{n}\right), \\
a_{n}=\frac{1}{2 \pi i} \int_{|z|=R} \frac{f(z)}{\left(z-\zeta_{1}\right) \cdots\left(z-\varsigma_{n+1}\right)} d z, \quad R>r .
\end{gathered}
$$

We define

$$
\begin{gathered}
M(R, f)=\max _{|z|=R}|f(z)|, \\
\mu(R, f)=\max _{n} \max _{|z|=R}\left|a_{n}\right|\left|z-\varsigma_{1}\right|\left|z-\varsigma_{2}\right| \cdots\left|z-\varsigma_{n}\right| .
\end{gathered}
$$

If $f(z)$ has finite order, then

$$
\log \mu(R, f) \leq \log M(R, f) \leq \log \mu(R, f)+O(\log R)
$$

(see $[\mathbf{4}, 1.11]$ ). This enables us to use $\mu(R, f)$ and $M(R, f)$ interchangeably in what follows.

LEMMA 2.1. Suppose $\phi(x)$ is a twice differentiable function such that $x \phi^{\prime}(x)$ increases to $\infty$ for large $x$. Suppose further that $\lim _{x \rightarrow \infty}(\log \phi(x)) /(\log x)=\rho$. Define $p(t)$ by $p(t) \phi^{\prime}(p(t))=t$ for all large $t$. Then if $f(z)$ is entire of order $\rho$ and

$$
\log M(R, f) \leq \phi(R),
$$


there exists a constant $\delta_{1}<1$ so that

$$
\log \left|a_{n}\right| \leq \phi(p(n))-n \log p(n)+O\left(n^{\delta_{1}}\right) .
$$

PrOOF. L'Hospital's Rule implies $\log p(t) / \log t \rightarrow 1 / \rho$. From (2.1) we have

$$
\left|a_{n}\right| \leq \frac{1}{2 \pi}\left\|\int_{|z|=R} \frac{f(z)}{\left(z-\zeta_{1}\right) \cdots\left(z-\zeta_{n+1}\right)} d z\right\| \leq R M(R, f)(R-r)^{-(n+1)} .
$$

Hence

$$
\log \left|a_{n}\right| \leq \phi(R)-n \log R+O(n / R) .
$$

Setting $R=p(n)$, we get (2.4).

LEMMA 2.2. Suppose $\psi(x)$ is a twice differentiable function such that $\psi^{\prime}(x)$ decreases to $-\infty$ for large $x$. Suppose also that $\lim _{x \rightarrow \infty}(\psi(x)) /(x \log x)=-1 / \rho$. Let $q(t)$ satisfy $\psi^{\prime}(q(t))=-\log t$. Then if $f(z)$ is entire of order $\rho$ and

$$
\log \left|a_{n}\right| \leq \psi(n)
$$

there exists a constant $\delta_{2}<\rho$ so that

$$
\log M(R, f) \leq \psi(q(R))+q(R) \log R+O\left(R^{\delta_{2}}\right) .
$$

ProOF. The conditions on $\psi$ imply that $\log q(t) / \log t \rightarrow \rho$. We have

$$
\log \mu(R, f) \leq \log \max _{n}\left|a_{n}\right|(R+r)^{n} \leq \max _{n}\left(\psi(n)+n \log R+\frac{n r}{R}\right) .
$$

A straightforward computation now yields

$$
\log M(R, f) \leq \psi(q(R))+q(R) \log R+O\left(\frac{q(R) \log R}{R}\right)
$$

which implies (2.7).

We now combine Lemmas 2.1 and 2.2 .

LEMMA 2.3. (i) Let $\phi$ satisfy the hypotheses of Lemma 2.1. Define $\psi(t)=$ $\phi(p(t))-t \log p(t)$. Then there exists a constant $\delta_{2}<\rho$ so that

$$
\log M(R, f) \leq \phi(R)+O\left(R^{\delta_{2}}\right)
$$

if and only if there exists a $\delta_{1}<1$ so that

$$
\log \left|a_{n}\right| \leq \psi(n)+O\left(n^{\delta_{1}}\right) .
$$

(ii) On the other hand, let $\psi$ satisfy the hypotheses of Lemma 2.2. Define $\phi(t)=$ $\psi(q(t))+q(t) \log t$. Then there exists a $\delta_{1}<1$ so that

$$
\log \left|a_{n}\right| \leq \psi(n)+O\left(n^{\delta_{1}}\right)
$$

if and only if there exists a $\delta_{2}<\rho$ so that

$$
\log M(R, f) \leq \phi(R)+O\left(R^{\delta_{2}}\right) .
$$

ProOF. To prove (i), observe that $\psi$ satisfies the conditions in Lemma 2.2 and

$$
\psi^{\prime}(t)=\left(\phi^{\prime}(p(t))-t / p(t)\right) p^{\prime}(t)-\log p(t)=-\log p(t) .
$$

Thus $p(q(t))=t$, and $\psi(q(t))+q(t) \log t=\phi(t)$. Therefore, by Lemmas 2.1 and 2.2 , we are done. The proof of (ii) is analogous. 
We say that the coefficients $a_{n}$ in $(2.1)$ have growth rate $\left[1 / \rho ; \tau_{0}, \ldots, \tau_{k}\right]$ if for every $\varepsilon>0$, we have

$$
\log \left|a_{n}\right|<-\frac{1}{\rho} n \log n+n\left(\tau_{0}+\frac{\tau_{1}}{\log n}+\cdots+\frac{\tau_{k}+\varepsilon}{(\log n)^{k}}\right)
$$

for all large $n$, and

$$
\log \left|a_{n}\right|>-\frac{1}{\rho} n \log n+n\left(\tau_{0}+\frac{\tau_{1}}{\log n}+\cdots+\frac{\tau_{k}-\varepsilon}{(\log n)^{k}}\right)
$$

for arbitrarily large $n$.

We order these growth rates lexicographically, and define arithmetic limits in the obvious way.

THEOREM 2.4. The entire function $f(z)$ given by $(2.1)$ has growth rate $\left(\rho ; \sigma_{0}\right.$, $\left.\sigma_{1}, \ldots, \sigma_{k}\right)$ if and only if the coefficients $a_{n}$ have growth rate $\left[1 / \rho ; \tau_{0}, \tau_{1}, \ldots, \tau_{k}\right]$, where the relation between $\sigma_{0}, \sigma_{1}, \ldots, \sigma_{k}$ and $\tau_{0}, \tau_{1}, \ldots, \tau_{k}$ is determined by the relation between the functions $\phi(R)$ and $\psi(t)$ in Lemma 2.3 .

PrOOF. We apply the first half of Lemma 2.3 to the function

$$
\phi(R)=R^{\rho}\left(\sigma_{0}+\sigma_{1} / \log R+\cdots+\sigma_{k} /(\log R)^{k}\right) .
$$

Let $R_{t}=p(t)$. Using this function in Lemma 2.1 , we easily see that $R_{t}^{\rho} /\left(\log R_{t}\right)^{k+1}$ $=O\left(t /(\log t)^{k+1}\right)$. Hence,

$$
R_{t}^{\rho}\left(\lambda_{0}+\lambda_{1} / \log R_{t}+\cdots+\lambda_{k} /\left(\log R_{t}\right)^{k}\right)=t+O\left(t /(\log t)^{k+1}\right)
$$

where $\lambda_{i}=\rho \sigma_{i}-(i-1) \sigma_{i-1} ; i=0,1, \ldots, k\left(\sigma_{-1}=0\right)$. Inverting (2.15) we get constants $\mu_{0}, \ldots, \mu_{k}$ such that

$$
R_{t}^{\rho}=t\left(\mu_{0}+\mu_{1} / \log t+\cdots+\mu_{k} /(\log t)^{k}\right)+O\left(t /(\log t)^{k+1}\right) .
$$

Taking logarithms in (2.16) we get constants $\nu_{0}, \ldots, \nu_{k}$ such that

$$
\log R_{t}=\frac{1}{\rho} \log t+\nu_{0}+\nu_{1} / \log t+\cdots+\nu_{k} /(\log t)^{k}+O\left(1 /(\log t)^{k+1}\right) .
$$

Using Lemma 2.3, we get the desired form for $\psi(t)$. The converse computation of $\phi(R)$ from $\psi(t)$ is entirely analogous.

The actual computation of $\tau_{0}, \ldots, \tau_{k}$ in terms of $\rho, \sigma_{0}, \ldots, \sigma_{k}$ is rather cumbersome. We will carry it one step beyond the known fact

$$
\tau_{0}=\frac{1}{\rho} \log \left(e \rho \sigma_{0}\right)
$$

Setting $\phi(R)=R^{\rho}\left(\sigma_{0}+\sigma_{1} / \log R\right)$ we get

$$
t=R_{t} \phi^{\prime}\left(R_{t}\right)=\rho \phi\left(R_{t}\right)+O\left(R_{t}^{\rho} /\left(\log R_{t}\right)^{2}\right) .
$$

Hence

$$
\begin{gathered}
\log t=\rho \log R_{t}+\log \left(\sigma_{0} \rho\right)+\frac{\sigma_{1} / \sigma_{0}}{\log R_{t}}+O\left(\frac{1}{\left(\log R_{t}\right)^{2}}\right), \\
\log R_{t}=\frac{1}{\rho} \log t-\frac{1}{\rho} \log \left(\rho \sigma_{0}\right)-\frac{\sigma_{1} / \sigma_{0}}{\log t}+O\left(\frac{1}{(\log t)^{2}}\right),
\end{gathered}
$$


and

$$
\begin{aligned}
\psi(t) & =\phi\left(R_{t}\right)-t \log R_{t} \\
& =-\frac{1}{\rho} t \log t+t\left(\frac{1}{\rho} \log \left(\sigma_{0} \rho e\right)+\frac{\sigma_{1} / \sigma_{0}}{\log t}+O\left(\frac{1}{(\log t)^{2}}\right)\right) .
\end{aligned}
$$

Thus

$$
\tau_{1}=\sigma_{1} / \sigma_{0} \text {. }
$$

3. The arithmetic limits. We expand the function $f \in \mathcal{F}$ in a generalized Taylor series at the points $\zeta_{1}, \zeta_{2}, \ldots$, where all $\zeta_{n} \in\left\{z_{1}, z_{2}, \ldots, z_{s}\right\}$. Thus

$$
f(z)=\sum_{n=0}^{\infty} a_{n}\left(z-z_{1}\right)^{n_{1}} \cdots\left(z-z_{s}\right)^{n_{s}}
$$

where $n_{i}=n_{i}(n)$ is a nondecreasing function of $n$ and $n_{1}+n_{2}+\cdots+n_{s}=n$. We further choose the exponents $n_{i}$ so that the quantities

$$
\Psi_{i}(n)=n_{i} ! \prod_{\substack{j=1 \\ j \neq i}}^{s}\left(z_{i}-z_{j}\right)^{n_{j}}, \quad i=1,2, \ldots, s,
$$

are nearly equal in absolute value.

We define $\psi_{i}(t)$ by

$$
-\psi_{i}(t)=\log \left|\Psi_{i}(t)\right|=\log \Gamma\left(t_{i}+1\right)+\sum_{j \neq i} t_{j} \log \left|z_{i}-z_{j}\right|,
$$

for $i=1,2, \ldots, s$, where $t=t_{1}+\cdots+t_{s}$. For a given $t$, we want to choose $t_{1}, \ldots, t_{s}$ in such a way that $\psi_{i}(t)$ does not depend on $i$, that is

$$
\psi_{1}(t)=\cdots=\psi_{s}(t), \quad t_{1}+t_{2}+\cdots+t_{s}=t .
$$

Let us call $\psi(t)$ the common value of $\psi_{i}(t)$ so obtained; thus

$$
-\psi(t)=\log \Gamma\left(t_{i}+1\right)+\sum_{j \neq i} t_{j} \log \left|z_{i}-z_{j}\right|, \quad i=1,2, \ldots, s .
$$

The solution of (3.2) leads to asymptotic expansions of the form

$$
t_{i}=\frac{1}{s} t\left(1+\frac{\gamma_{i 1}}{\log t}+\frac{\gamma_{i 2}}{(\log t)^{2}}+\cdots+\frac{\gamma_{i k}}{(\log t)^{k}}\right)+O\left(\frac{t}{(\log t)^{k+1}}\right),
$$

where $\sum_{i=1}^{s} \gamma_{i j}=0, j=1,2, \ldots, k$, and

$$
\psi(t)=-\frac{1}{s} t \log t+\frac{1}{s} t\left(\gamma_{0}+\frac{\gamma_{1}}{\log t}+\cdots+\frac{\gamma_{k}}{(\log t)^{k}}\right)+O\left(\frac{t}{(\log t)^{k+1}}\right) .
$$

Let $P(z)=\left(z-z_{1}\right) \cdots\left(z-z_{s}\right)$. If we compare terms of order $t$, then (3.2) yields

$$
\begin{aligned}
\gamma_{0} & =1+\log s-\gamma_{i 1}-\sum_{j \neq i} \log \left|z_{i}-z_{j}\right| \\
& =1+\log s-\gamma_{i 1}-\log \left|P^{\prime}\left(z_{i}\right)\right| .
\end{aligned}
$$

Summing over $i$, we get

$$
\gamma_{0}=1+\log s-2 \log \prod_{1 \leq i<j \leq s}\left|z_{i}-z_{j}\right|=1+\log s-2 \log V
$$


where $V$ is the absolute value of the Vandermonde of $z_{1}, \ldots, z_{s}$. Thus (3.5) yields

$$
\gamma_{i 1}=\log \left|V^{2} / P^{\prime}\left(z_{i}\right)\right|
$$

We now compare terms of order $t / \log t$ to get

$$
\begin{aligned}
\gamma_{1}= & -\gamma_{i 2}+\gamma_{i 1} \log s-\sum_{j \neq i} \gamma_{j 1} \log \left|z_{i}-z_{j}\right| \\
= & -\gamma_{i 2}+2 \log V \log s-\log \left|P^{\prime}\left(z_{i}\right)\right| \log s-2 \log V \log \left|P^{\prime}\left(z_{i}\right)\right| \\
& +\sum_{j \neq i} \log \left|z_{i}-z_{j}\right| \log \left|P^{\prime}\left(z_{i}\right)\right| .
\end{aligned}
$$

Summing over $i$, we obtain

$$
\begin{aligned}
\gamma_{1}= & \left(2-\frac{2}{s}\right) \log V \log s-\frac{4}{s}(\log V)^{2} \\
& +\frac{1}{s} \sum_{i=1}^{s} \sum_{j \neq i} \log \left|z_{i}-z_{j}\right| \log \left|P^{\prime}\left(z_{i}\right)\right| .
\end{aligned}
$$

Hence

$$
\begin{aligned}
\gamma_{i 2}= & \frac{2}{s} \log V \log s+\frac{4}{s}(\log V)^{2}-\frac{1}{s} \sum_{i=1}^{s} \sum_{j \neq i} \log \left|z_{i}-z_{j}\right| \log \left|P^{\prime}\left(z_{i}\right)\right| \\
& -\log \left|P^{\prime}\left(z_{i}\right)\right| \log \left(s V^{2}\right)+\sum_{j \neq i} \log \left|z_{i}-z_{j}\right| \log \left|P^{\prime}\left(z_{i}\right)\right| .
\end{aligned}
$$

In an analogous manner we can compute the constants $\gamma_{i j}$ for $i=1,2, \ldots, s$, $j=3, \ldots, k$.

We now choose the integers $n_{i}$ to satisfy

$$
n_{i}(n)=t_{i}(n)+O(1) \text {. }
$$

To do this, we let $\zeta_{n}$ be the $z_{i}$ of lowest index for which $t_{i}(n)-n_{i}(n-1)$ is maximal.

THEOREM 3.1. The arithmetic limit for the generalized Taylor coefficients of functions in $\mathcal{F}$ is $\left[1 / s ; \gamma_{0} / s, \ldots, \gamma_{k} / s\right]$, and the corresponding arithmetic limit for the growth rates of these functions is $\left(s ; \sigma_{0}, \sigma_{1}, \ldots, \sigma_{k}\right)$, where the relation between the $\sigma_{i}$ and the $\tau_{i}=\gamma_{i} / s$ is as in Theorem 2.4 .

ProOF. Let $f(z) \in \mathcal{F}$ have generalized Taylor expansion

$$
f(z)=\sum_{k=0}^{\infty} a_{n}\left(z-z_{1}\right)^{n_{1}} \cdots\left(z-z_{s}\right)^{n_{s}},
$$

where the $n_{i}$ satisfy (3.11). Assume that $\zeta_{n+1}=z_{i}$, that is $n_{i}(n+1)=n_{i}(n)+1$. Then (3.12) yields

$$
f^{\left(n_{i}\right)}\left(z_{i}\right)=a_{n} \Psi_{i}(n)+R_{n},
$$

where the remainder $R_{n}$ depends only on the coefficients $a_{0}, \ldots, a_{n-1}$.

If the growth rate of the $a_{n}$ is less than $\left[1 / s ; \gamma_{0} / s, \ldots, \gamma_{k} / s\right]$, then we must have

$$
\log \left|f^{\left(n_{i}\right)}\left(z_{i}\right)-R_{n}\right|=\log \left|a_{n}\right|-\psi_{i}(n) \rightarrow-\infty \quad \text { as } n \rightarrow \infty .
$$


Since any two distinct integers of $K$ have difference at least 1 in absolute value, there is at most one integral value $f^{\left(n_{i}\right)}\left(z_{i}\right)$ which makes the left side of $(3.14)$ less than $\log \frac{1}{2}$ for a given $R_{n}$. Thus when $n$ is sufficiently large, the coefficients $a_{0}, a_{1}, \ldots, a_{n-1}$ determine $f^{\left(n_{i}\right)}\left(z_{i}\right)$, and hence $a_{n}$, uniquely. This shows that there can only be a countable number of such $f(z)$.

On the other hand, there is a constant $c$ such that every circle of radius $c$ contains at least two integers of $K$. Thus if we permit $\left|f^{\left(n_{i}\right)}\left(z_{i}\right)-R_{n}\right| \geq c$ for all $n$, we get at least two possible choices for $f^{\left(n_{i}\right)}\left(z_{i}\right)$. Hence we get an uncountable set of functions in $₹$.

REMARK. If $s=1$, the remainder $R_{n}$ in (3.13) is 0 for all $n$. Hence if the growth of $f(z)$ is below the arithmetic limit $\left(1, \sigma_{0}, \sigma_{1}, \ldots, \sigma_{k}\right)$, then $f(z)$ is a polynomial.

4. Functions in $₹$ with growth rate below the arithmetic limit. It is known [3] that a function $f(z) \in \mathcal{F}$ of order $\rho<s$ satisfies a linear differential equation with $K$-integral coefficients. For more general growth rates, the situation is as follows.

THEOREM 4.1. Let $F(z) \in \mathcal{F}$ have growth rate less than the arithmetic limit $\left(s ; \sigma_{0}, \ldots, \sigma_{k}\right)$. If there exists a nonconstant function $f(z) \in \mathcal{F}$ of order $<s$, then $F(z)$ satisfies a linear differential equation with coefficients in $K[f]$, the ring of polynomials in $f$ with coefficients in $K$.

REMARK. If $s=1, F(z)$ is a polynomial by the remark following Theorem 3.1. Hence it satisfies the equation $F^{(n)} \equiv 0$ for some $n$. Thus we may assume from now on that $s \geq 2$.

For the proof of Theorem 4.1 we need an estimate for the derivatives of entire functions.

LEMMA 4.2. For any entire function $f$ and any $r>0$, we have

$$
M\left(R, f^{(n)}\right) \leq r^{-n} M(R+n r, f) .
$$

Proof. By Cauchy's formula we have

$$
f^{\prime}(z)=\frac{1}{2 \pi i} \int_{|\zeta-z|=r} \frac{f(\zeta) d \zeta}{(\zeta-z)^{2}}
$$

and hence

$$
M\left(R, f^{\prime}\right) \leq r^{-1} M(R+r, f) .
$$

Iterating (4.2) $n$ times, we get (4.1).

ProOF OF THEOREM 4.1. Suppose $F(z) \in \mp$ and

$$
M(R, F)<R^{s}\left(\sigma_{0}+\frac{\sigma_{1}}{\log R}+\cdots+\frac{\sigma_{k}-\delta}{(\log R)^{k}}\right)
$$

for some $\delta>0$ and $R$ sufficiently large. We construct a function

$$
\Phi(z)=\sum_{u=0}^{U} \sum_{v=0}^{V} \lambda_{u v} f(z)^{u} F^{(v)}(z),
$$

where the $\lambda_{u v}$ are rational integers, not all zero. 
Let $m$ be a positive integer to be chosen sufficiently large later, and set

$$
U=\left[m^{1-1 / s} /(\log m)^{k+2}\right], \quad V=\left[m^{1 / s}(\log m)^{2 k+4}\right] .
$$

We expand $\Phi$ in the generalized Taylor series given by (3.11), (3.12), and choose the $\lambda_{u v}$ so that

$$
\Phi^{\left(\nu_{i}\right)}\left(z_{i}\right)=0, \quad \nu_{i}=0,1, \ldots, n_{i}(m) ; i=1,2, \ldots, s .
$$

This is a system of $\left(n_{1}(m)+1\right)+\left(n_{2}(m)+1\right)+\cdots+\left(n_{s}(m)+1\right)=m+s$ linear equations with $K$-integral coefficients for the $(U+1)(V+1)$ unknowns $\lambda_{u v}$. If $K \neq \mathbf{Q}$ we take rational and irrational parts, getting $2 m+2 s$ equations with coefficients in $\frac{1}{2} \mathbf{Z}$. These coefficients are rational or irrational parts of

$$
\begin{aligned}
C_{i, \nu_{i}, u, v} & =\left.\frac{d^{\nu_{i}}}{d z^{\nu_{i}}}\left(f(z)^{u} F^{(v)}(z)\right)\right|_{z=z_{i}} \\
& =\left.\sum_{k=0}^{\nu_{i}}\left(\frac{\nu_{i}}{k}\right) \frac{d^{k}}{d z^{k}}\left(f(z)^{u}\right)\right|_{z=z_{i}} F^{\left(v+\nu_{i}-k\right)}\left(z_{i}\right) .
\end{aligned}
$$

By Lemma 4.2 , with $R=\max _{1 \leq i \leq s}\left|z_{i}\right|$ and $r=1 / m$, we have

$$
\begin{aligned}
\left|C_{i, \nu_{i}, u, v}\right| \leq & 2^{n_{i}(m)} m^{n_{i}(m)} M\left(R+n_{i}(m) / m, f^{U}\right) \\
& \times m^{V+n_{i}(m)} M\left(R+\left(V+n_{i}(m)\right) / m, F\right) .
\end{aligned}
$$

From $(3.10)$ and $(3.3)$ we see that $n_{i}(m)<m(1 / s+\varepsilon)$ for large $m$. Hence for some fixed $C>1$,

$$
\begin{gathered}
m^{n_{i}(m)} \leq m^{V+n_{i}(m)} \leq C^{m \log m}, \\
M\left(R+n_{i}(m) / m, f^{U}\right)<C^{m}, \\
M\left(R+\left(V+n_{i}(m) / m\right), F\right)<C .
\end{gathered}
$$

Thus (4.6) yields

$$
\left|C_{i, \nu_{i}, u, v}\right|<C^{m \log m} .
$$

The number of unknowns is $(U+1)(V+1)>m(\log m)^{k+2}>2 m+2 s$. Hence by Siegel's lemma (see $[\mathbf{1}]$ ), we can find rational integers $\lambda_{u v}$, not all zero, satisfying (4.5) and

$$
\left|\lambda_{u v}\right| \leq\left(C_{1}^{m \log m}\right)^{(2 m+2 s) /\left(m(\log m)^{k+2}-(2 m+2 s)\right)}<C_{2}^{m /(\log m)^{k+1}} .
$$

We now show that if $m$ is sufficiently large, then $\Phi(z)$ vanishes identically. From (4.5) we have $a_{0}=a_{1}=\cdots=a_{m}=0$.

Let $i$ be the index with $n_{i}(m+2)=n_{i}(m+1)+1$. It suffices to show that $\Phi^{n_{i}(m+1)}\left(z_{i}\right)=0$ if $m$ is sufficiently large. For this implies that $a_{m+1}=0$, and the result follows by induction.

We have

$$
\begin{aligned}
\Phi^{n_{i}(m+1)}\left(z_{i}\right) & =a_{m+1} \Psi_{i}(m+1) \\
& =\Psi_{i}(m+1) \frac{1}{2 \pi i} \int_{|z|=R} \frac{\Phi(z) d z}{\prod_{j=1}^{s}\left(z-z_{j}\right)^{n_{j}(m+1)}\left(z-z_{i}\right)} .
\end{aligned}
$$


Thus

(4.9) $\log \left|\Phi^{n_{i}(m+1)}\left(z_{i}\right)\right| \leq-\psi_{i}(m+1)+\log M(R, \phi)-(m+1) \log R+(C m / R)$.

We need only show that for sufficiently large $M$ the right side of (4.9) is negative. Now

$$
\begin{aligned}
\log M(R, \Phi) \leq & \log (U+1)+\log (V+1)+\log \max \left|\lambda_{u v}\right| \\
& +U \log M(R, f)+\max _{v \leq V} \log M\left(R, F^{(v)}\right) \\
\leq & C m /(\log m)^{k+1}+C R m^{1-1 / s} /(\log m)^{k+2} \\
& -V \log r+\log M(R+V r, F) .
\end{aligned}
$$

Setting $r=1 / V$ and $L=\log R$, we obtain

$$
\begin{aligned}
\log M(R, \Phi) \leq & C m(\log m)^{k+1}+C R m^{1-1 / s}(\log m)^{k+2} \\
& +R^{s}\left(\sigma_{0}+\frac{\sigma_{1}}{L}+\cdots+\frac{\sigma_{k}-\delta}{L^{k}}\right)+C R^{s-1} .
\end{aligned}
$$

Substituting (4.11) in (4.9), we get

$$
\begin{aligned}
\log \left|\Phi^{n_{i}(m+1)}\left(z_{i}\right)\right| & \leq\left\{\frac{1}{s} m \log m-\frac{1}{s} m\left(\gamma_{0}+\frac{\gamma_{1}}{\log m}+\cdots+\frac{\gamma_{k}}{(\log m)^{k}}\right)\right. \\
& \left.+R^{s}\left(\sigma_{0}+\frac{\sigma_{1}}{L}+\cdots+\frac{\sigma_{k}}{L^{k}}\right)-m \log R\right\} \\
& +C m / R-\log R+C R^{s-1}+C m /(\log m)^{k+1} \\
& +C R m^{1-1 / s} /(\log m)^{k+2}-\frac{\delta R^{s}}{(\log R)^{k}}+O\left(\frac{m}{(\log m)^{k+1}}\right) .
\end{aligned}
$$

We now choose $R=p(m)$ as in Lemma 2.1. By Theorem 2.4, the quantity in braces is $O\left(m /(\log m)^{k+1}\right)$. Since $R=O\left(m^{1 / s}\right)$, the term $-\delta R^{s} /(\log R)^{k}$ dominates, and the limit is $-\infty$. This completes the proof.

5. Solutions of differential equations with periodic coefficients. We continue to assume in this section that there is a nonconstant function $f \in \mathcal{F}$ of order $<s$. It was shown in $[\mathbf{3}]$ and $[\mathbf{1 1}]$ that $f$ is either a polynomial or an exponential polynomial. In the latter case $f$ is clearly periodic.

By Theorem 4.1, every function $F \in \mathcal{F}$ with order $s>1$ and growth rate below the arithmetic limit $\left(s, \sigma_{0}, \sigma_{1}, \ldots, \sigma_{k}\right)$ satisfies a linear differential equation with coefficients in $K[f]$.

We now discuss the case where $f(z)$ is periodic, say with period $\omega$. The finction $F(z)$ cannot be periodic, since it would then be infinitely integer valued at more than $s$ points - in fact, at infinitely many points $z_{i}+n \omega$. This would imply that $F(z)$ is of order $\leq 1$, contrary to assumption.

LEMMA 5.1. Let $F(z)$ be entire and satisfy the differential equation

$$
A_{0}(z) F+A_{1}(z) F^{\prime}+\cdots+A_{n}(z) F^{(n)}=0,
$$

where $A_{i}(z+\omega)=A_{i}(z), 0 \leq i \leq n$. Then $F(z)$ satisfies a linear difference equation

$$
C_{0} F(z)+C_{1} F(z+\omega)+\cdots+C_{p} F(z+p \omega)=0
$$

with constant coefficients $C_{0}, C_{1}, \ldots, C_{p} ; C_{p} \neq 0$. 
ProOF. The functions $F(z+k \omega), 0 \leq k \leq n$, are all solutions of (5.1); hence they are linearly dependent.

Let the zeros of the characteristic polynomial $C_{0}+C_{1} t+\cdots+C_{p} t^{p}$ of (5.2) be $e^{\lambda_{1}}, e^{\lambda_{2}}, \ldots, e^{\lambda_{k}}$, with multiplicities $M_{1}+1, \ldots, M_{k}+1$. Then for all integers $q$, we have

$$
F(z+q \omega)=\sum_{i=1}^{k}\left(a_{i 0}(z)+a_{i 1}(z) q+\cdots+a_{i M_{i}}(z) q^{M_{i}}\right) e^{\lambda_{i} q}
$$

(see, for example, [5, p. 384]). Here the $a_{i j}(z)$ are entire functions which satisfy

$$
\sum_{j=0}^{M_{i}} a_{i j}(z+\omega) q^{j}=e^{\lambda_{i}} \sum_{j=0}^{M_{i}} a_{i j}(z)(q+1)^{j} .
$$

If we set $b_{i j}(z)=a_{i j}(z) e^{-\lambda_{i} z / \omega}$, then (5.4) becomes

$$
\sum_{j=0}^{M_{i}} b_{i j}(z+\omega) q^{j}=\sum_{j=0}^{M_{i}} b_{i j}(z)(q+1)^{j} .
$$

In particular, this shows that $b_{i 0}(z)=\sum_{j=0}^{M_{i}} c_{i j}(z) z^{j}$, where $c_{i j}(z+\omega)=c_{i j}(z)$. From (5.3) with $q=0$ we get

$$
F(z)=\sum_{i=1}^{k} \sum_{j=0}^{M_{i}} c_{i j}(z) z^{i} e^{\lambda_{i} z / \omega} .
$$

LEMmA 5.2. Let $e^{\lambda_{i}}, i=1,2,3, \ldots$, be distinct. Then the functions $z^{j} e^{\lambda_{i} z / \omega}$, $i=1,2,3, \ldots ; j=0,1,2, \ldots$, are linearly independent over the set of functions of period $\omega$. with

Proof. Assume not. Then there exist functions $F_{i j}(z)$ of period $\omega$, not all zero,

$$
\sum_{i=1}^{n} \sum_{j=0}^{m} F_{i j}(z) z^{j} e^{\lambda_{i} z / \omega}=0
$$

We may assume that $m$ is minimal and that the number of nonzero $F_{i m}(z)$ is minimal. Collecting the nonzero terms of (5.7) with $j=m$, we obtain

$$
z^{m}\left(F_{n_{1} m}(z) e^{\lambda_{n_{1}} z / \omega}+\cdots+F_{n_{k} m}(z) e^{\lambda_{n_{k}} z / \omega}\right),
$$

where the $F_{n_{i} m}(z)$ are nonzero. Substitution of $z+\omega$ into (5.7) gives an equation with corresponding term

$$
z^{m}\left(F_{n_{1} m}(z) e^{\lambda_{n_{1}} z / \omega} e^{\lambda_{n_{1}}}+\cdots+F_{n_{k} m}(z) e^{\lambda_{n_{k}} z / \omega} e^{\lambda_{n_{k}}}\right) .
$$

Since the $e^{\lambda_{n_{i}}}$ are distinct and $k$ is minimal, this implies that $k=1$. Thus we may assume that the highest terms of (5.7) are

$$
z^{m-1}\left(F_{p_{1} m-1}(z) e^{\lambda_{p_{1}} z / \omega}+\cdots+F_{p_{r} m-1}(z) e^{\lambda_{p_{r}} z / \omega}\right)+z^{m} F(z) e^{\lambda z / \omega},
$$

where the $F_{p_{k} m-1}(z)$ and $F(z)$ are nonzero, and $\lambda=\lambda_{i}$ for some $i$. 
Substituting $z+\omega$ into (5.7), we get an equation with highest terms

$$
\begin{array}{r}
z^{m-1}\left(F_{p_{1} m-1}(z) e^{\lambda_{p_{1}} z / \omega} e^{\lambda_{p_{1}}}+\cdots+F_{p_{r} m-1}(z) e^{\lambda_{p_{r}} z / \omega} e^{\lambda_{p_{r}}}\right. \\
\left.+m \omega F(z) e^{\lambda z / \omega} e^{\lambda}\right)+z^{m} F(z) e^{\lambda z / \omega} e^{\lambda .}
\end{array}
$$

Since $m$ is minimal we must have $m \omega F(z) e^{\lambda z / \omega} e^{\lambda}=0$, which implies $m=0$. Thus (5.7) reduces to $F(z) e^{\lambda z / \omega}=0$, which is impossible.

LEMMA 5.3. Let $F(z)$ be analytic in $0<|z|<\infty$ with Laurent expansion

$$
F(z)=\sum_{n=-\infty}^{\infty} a_{n} z^{n}
$$

Suppose that both $f(z)=\sum_{n=0}^{\infty} a_{n} z^{n}$ and $g(z)=\sum_{n=1}^{\infty} a_{-n} z^{n}$ have order 0 . If $F(z)$ satisfies a linear differential equation with rational functions as coefficients, then it is a Laurent polynomial.

PROOF. By clearing denominators, we may assure that the coefficients of the differential equation are polynomials. We wish to show that $a_{n}=0$ for all large $|n|$ in (5.8). Since the conditions are symmetric in $z$ and $1 / z$, it suffices to prove the result for large positive $n$. The hypothesis implies that

$$
\log \left|a_{n}\right| / n \log n \rightarrow-\infty,
$$

where we take $\log 0$ to mean $-\infty$. Thus either $a_{n}=0$ for all sufficiently large $n$, or for every $M>0$ there is an infinite sequence of indices $n_{1}, n_{2}, \ldots$ with

$$
\left|a_{n_{i}+p}\right| \leq\left|a_{n_{i}}\right| / n_{i}^{p M}, \quad p=0,1,2, \ldots
$$

Now consider the differential equation satisfied by $F(z)$ :

$$
\left(b_{00}+b_{01} z+\cdots+b_{0 k_{0}} z^{k_{0}}\right) F+\cdots+\left(b_{l 0}+b_{l 1} z+\cdots+b_{l k_{l}} z^{k_{l}}\right) F^{(l)}=0 .
$$

We substitute the Laurent series (5.8) into (5.10) and set the coefficient of $z^{n}$ equal to 0 . This gives a system of linear equations $E_{n}$ for the $a_{i}$. If $k=\operatorname{Max}_{j}\left(k_{j}-j\right)$ and $n=k+\nu$, then $a_{0}, a_{1}, \ldots, a_{\nu-1}$ do not appear in equation $E_{n}$. Consider $E_{k+n_{i}}$, where $n_{i}$ satisfies (5.9). The coefficient of $a_{n_{i}}$ in $E_{k+n_{i}}$ is

$$
B_{n_{i}}=\sum_{j}^{\prime} b_{j k_{j}} n_{i} ! /\left(n_{i}-j\right) !
$$

where the summation is extended over all $j$ for which $k_{j}-j=k$. It is clear that $B_{n_{i}}$ is nonzero for all but a finite number of indices $i$. Moreover there exists a positive $B$ such that $\left|B_{n_{i}}\right|>B$ for all large $i$. On the other hand, the coefficients of all terms $a_{n_{i}+p}$ in $E_{k+n_{i}}$ are bounded by $C_{n_{i}}^{l}$. We choose $M>l$ in (5.9). Then for all large $n_{i}$, the contribution of the terms $a_{n_{i}+p}(p>0)$ is too small to cancel the term $B_{n_{i}} a_{n_{i}}$. This contradiction shows that $f(z)$ and $g(z)$ are polynomials, so $F(z)$ is a Laurent polynomial.

We now apply these lemmas to the case where the $A_{i}(z)$ in (5.1) are exponential polynomials, i.e. Laurent polynomials in a single exponential. Substituting (5.6) into (5.1) and using Lemma 5.2, we get a system of equations

$$
\begin{aligned}
& L_{i j} c_{i j}(z)+L_{i j+1} c_{i j+1}(z)+\cdots+L_{i M_{i}} c_{i M_{i}}(z)=0, \\
& i=1,2, \ldots, k ; j=0,1, \ldots, M_{i},
\end{aligned}
$$


where the $L_{i j}$ are linear differential operators with exponential polynomial coefficients. Since the $C_{i j}(z)$ are entire of period $\omega$, they can be expressed as Laurent series in $w=e^{2 \pi i z / \omega}$ which satisfy the hypotheses of Lemma 5.3. Thus (5.12) with $j=M_{i}$ implies that the $C_{i M_{i}}$ satisfy linear differential equations with coefficients which are Laurent polynomials in $w$. Lemma 5.3 implies that the $C_{i M_{i}}$ are themselves exponential polynomials. Substituting these into (5.12) and using Lemma 5.3 again, we find that the $C_{i M_{i}-1}$ are exponential polynomials. Continuing in this way, we find that all the $C_{i j}$ are exponential polynomials. Hence, by (5.6) the order of $F(z)$ is $\leq 1$. Thus we have the following

THEOREM 5.4. If $\mathcal{F}$ contains nonconstant periodic functions of order $<s$, then all functions in $₹$ with growth rate below the arithmetic limit $\left(s ; \sigma_{0}, \ldots, \sigma_{k}\right)$ have order $\leq 1$.

\section{REFERENCES}

1. A. Baker, Transcendental number theory, Cambridge Univ. Press, London, 1975.

2. A. H. Cayford, A class of integer valued entire functions, Trans. Amer. Math. Soc. 141 (1969), 415-432.

3. A. Cayford and E. G. Straus, On differential rings of entire functions, Trans. Amer. Math. Soc. 209 (1975), 283-293.

4. B. Ja. Levin, Distribution of zeros of entire functions, Transl. Math. Monos., vol. 5, Amer. Math. Soc., Providence, R. I., 1964.

5. L. M. Milne-Thomson, The calculus of finite differences, Macmillan, New York, 1933.

6. L. D. Neidleman and E. G. Straus, Functions whose derivatives at a point form a finite set, Trans. Amer. Math. Soc. 140 (1969), 411-414.

7. D. Sato, Integer valued entire functions, Thesis, UCLA, 1961; see also Sugaku 14 (1962/63), 95-98 and 99-100.

8. D. Sato and E. G. Straus, On the rate of growth of Hurwitz functions of a complex or p-adic variable, J. Math. Soc. Japan 171 (1965), 17-29.

9. E. G. Straus, On entire functions with algebraic derivatives at certain algebraic points, Amer. J. Math. 52 (1950),188-198.

10. __ On polynomials whose derivatives have integer values at the integers, Proc. Amer. Math. Soc. 2 (1951), 24-27.

11. __ Differential rings of meromorphic functions, Acta Arith. 21 (1972), 271-284.

12. __ Differential rings of meromorphic functions of a non-Archimedean variable, Diophantine approximation and its applications (Proc. Conf. Washington, D. C., 1972), Academic Press, New York, 1973, pp. 295-308.

Department of Mathematics, University of CAlifornia, los Angeles, CaliFORNIA 90024 